\title{
Investigation of Test Outcomes Supported by Graphs
}

\author{
Sylvia Encheva ${ }^{1, *}$ \\ ${ }^{1}$ Stord/Haugesund University College, Bjørnsonsg. 45, 5528 Haugesund, Norway \\ ${ }^{*}$ Corresponding author
}

\begin{abstract}
Students' knowledge assessment has occupied the attention of many researches pertaining to different disciplines. Recent areas of interest in this regard include availability of preliminary skills and status of latest obtained knowledge, selfassessment, grading methods, tests, hints, help functions, etc. Involvement of smart technology in assessment processes is another important issue which rapidly gains popularity among students and lecturers. What seems to have received less attention in earlier studies is a way to establish whether apparent lack of knowledge is related to intrinsic or explicit terms and concepts. Application of selected graphs is put forward in this work in an attempt to increase the likelihood of an improved knowledge assessment.
\end{abstract}

Keywords-graphs; knowledge; assessment

\section{INTRODUCTION}

Assessment of students' knowledge is a very well discussed research area, [1], [15], [16], assisted by a large number of automated tests that have been developed last decades, [7] and [8]. Some of them aim at assessment of factual knowledge, others pay particular attention to detection of misunderstanding, misconception and misinterpretation of terms and general ideas inferred or derived from explicit instances, yet another of them are focusing on recognition of partially correct answers, and yet another of them try to prevent misuse of grading systems, [13].

The majority of automated tests provide feedback to both students and lectures about the amount of correct answers, a history of tests' results, as well as suggestions for further readings, examples, Socratic hints, etc., [16]. What seems to be less present is visualization of tests results emphasizing relations between lack of intrinsic and explicit knowledge. Visualization tools can support development of improved lectures by emphisizing those places where students experience problems in understanding and learning that subject. Our contribution for easier location of learning related obsticles is proposing employment of particular graphs and defeasible theory. The former is characterised by well structured connections between vertices which subsiquently contributes for building clear relationships among concepts. The main advantage of the latter is the combination of two desirable features: enhanced representational capabilities allowing one to reason with incomplete and contradictory information, coupled with low computational complexity compared to mainstream nonmonotonic reasoning, [2].

The rest of the paper is organized as follows. Related work and supporting theory may be found in Section 2. The obtained results are presented in Section 3. The paper ends with a conclusion in Section 4.

\section{RELATED WORK}

Graph related statements are taken mainly from [4] and [6]. The Petersen graph has ten vertices and fifteen edges, [9]. It appeared first in 1886 in a paper written by A. B. Kempe but carries the name of Julius Petersen presenting it in 1898. The Petersen family contains seven graphs that can be constructed from the Petersen graph.

Martin Kneser was the first one to discover essential features of Kneser graphs, [11]. A vertex in a Kneser graph $\mathrm{K}(\mathrm{n}, \mathrm{k})$ is a $k$-element subset of a set with cardinality $n$. Any vertex is adjacent to $\left(\begin{array}{c}n-k \\ k\end{array}\right)$ vertices where any two adjacent vertices stand for two disjoint sets.

An Odd graph is a Kneser graph with $\left(\begin{array}{c}2 n-1 \\ n-1\end{array}\right)$ vertices and $\frac{n\left(\begin{array}{c}2 n-1 \\ n-1\end{array}\right)}{2}$ edges. A vertex of the Odd graph can be seen as a subset with cardinality $n-1$ of a set with cardinality $2 n-1$.

Defeasible reasoning is a rule-based approach to reasoning with incomplete and inconsistent information, [2].

A defeasible theory is a triple $(F ; R ;>)$, where $F$ is a set of literals (called facts), $R$ a finite set of rules, and $>$ a superiority relation on $R$.

There are three kinds of rules:

Strict rules are denoted by $A \rightarrow p$, where $A$ is a finite set of literals and $p$ is a literal, and are interpreted in the classical sense: whenever the premises are indisputable (e.g. facts) then so is the conclusion.

Inferences from facts and strict rules only are called definite inferences. Facts and strict rules are intended to define relationships that are definitional in nature. Thus defeasible logics contain no mechanism for resolving inconsistencies in definite inference.

Defeasible rules are denoted by $A \Rightarrow p$, and can be defeated by contrary evidence.

Defeaters are used to prevent some conclusions. In other words, they are used to defeat some defeasible rules by producing evidence to the contrary. 
A superiority relation is an acyclic relation $>$ on $R$ (that is, the transitive closure of $>$ is irreflexive). Given two rules $r 1$ and $r 2$, if we have that $r 1>r 2$, then we will say that $r 1$ is superior to $r 2$, and $r 2$ inferior to $r 1$, [2]. This expresses that $r 1$ may override $r 2$.

Defeasible logic has attracted significant attention in nonmonotonic reasoning and found many applications, particularly in expert systems and knowledge-based systems communities, [14].

Two very interesting problems are considered in [4], namely the problem of determining a consensus from a group of orderings and the problem of making statistically significant statements about ordering.

Two elements $a$ and $b$ where $a \neq b$ and $a, b \in P$ are comparable if $a \leq b$ or $b \leq a$, and incomparable otherwise. If $\forall a, b$ where $a, b \in P$ are comparable, then $P$ is chain. If $\forall a, b$ where $a, b \in P$ are incomparable, then $P$ is antichain.

A relation $I$ is an indifference relation when given $A I B$ neither $A>B$ nor $A<B$ has place in the componentwise ordering. A partial ordering whose indifference relation is transitive is called a weak ordering.

Let $w_{1}, w_{2}, w_{3}$ be weak orderings. Then $w_{2}$ is between $w_{1}$ and $w_{3}$ if each decision made by $w_{2}$ is made by either $w_{1}$ or $w_{3}$ and any decision made by both $w_{1}$ and $w_{3}$ is made by $w_{2}$, i.e.

$$
w_{1} \cap w_{3} \subseteq w_{2} \subseteq w_{1} \cup w_{3} .
$$

The distance $d\left(w_{1}, w_{3}\right)$ is defined as

$$
d\left(w_{1}, w_{2}\right)+d\left(w_{2}, w_{3}\right)=d\left(w_{1}, w_{3}\right) .
$$

The distance is a metric in the usual sense, it is invariant under permutation of alternatives, and the minimum positive distance is 1 . Further more if two weak orderings agree except for a set of alternatives which is an open interval in both, then the distance may be computed as if the alternatives in this set were the only objects being ranked.

\section{INTRINSIC AND EXPLICIT KNOWLEDGE}

In knowledge assessment it is preeminent to find out where the problems in learning are situated. Of course the amount of correct responses has also its value but here we focus on discovering whether difficulties come from insufficient factual or explicit knowledge, or both.

Consider short tests of three questions. Such tests are suitable for quick self-assessment or being used in face to face teaching. Tests are associated with vertices of the graph in Figure 1 which is in fact a member of Petersen family graphs. Two vertices are connected when both of them contain a question addressing the same issue in a topic. A test associated with a vertex from the inner circle (in green) contains three questions related to explicit knowledge. A test associated with a vertex from the outer circle (in black) contains two questions related to intrinsic knowledge and one question related to explicit knowledge. If one of these questions receives a wrong answer it is an indication of a lack of knowledge, misconception or misinterpretation. The student is automatically suggested some examples clearing the matter, ascended by a different test where the same problem is addressed.

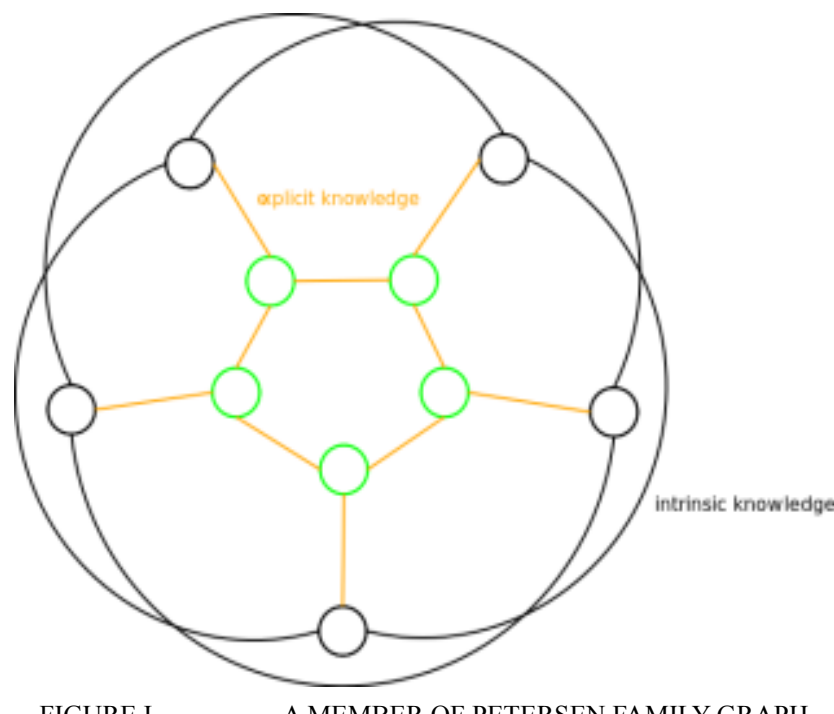

FIGURE I.

A MEMBER OF PETERSEN FAMILY GRAPH

A lecturer can follow closely where the majority of problems occur and act upon that either during a lecture or by introducing changes for the next course. Such actions involve providing hints, examples, additional reading, rephrasing questions as well as removing unclear questions and inserting new, hopefully better ones.

The idea in the graph in Figure 1 can be applied in case of a larger number of tests. A structure in Figure 2 is obtained from the graph in Figure 1 after extending the number of vertices by two. Additional vertices can be added in a similar manner.

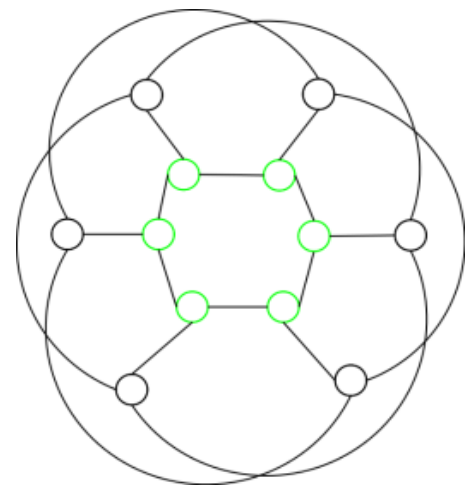

FIGURE II.

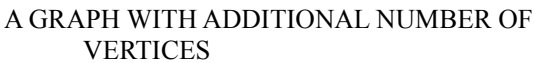

Vertices in the Odd graph in Figure 3 are placed on four circles where the most inner circle contains fourteen vertices and the three other circles contain seven vertices each. A vertex from the most inner circle (in green) is adjacent to one of the 
vertices in any of the four circles. Colours are used only to asist in distinguishing one circle from the next. All vertices situated on the three most outer circles are adjacent to two of the verteces on the most inner circle and to two of the verteces on the same circle where they are placed.

The Odd graph can be used for tests consisting of four questions. Thus vertices placed on the most inner circle correspond to tests with one intrinsic and three explicit questions while vertices placed on the other three circles correspond to tests with two intrinsic and two explicit questions. The rest is analogous to the case where a member of Petersen family graph is discussed.

Numbers associated with vertices correspond to different pulls of questions and thus avoiding repetition of questions in conciqutive tests.

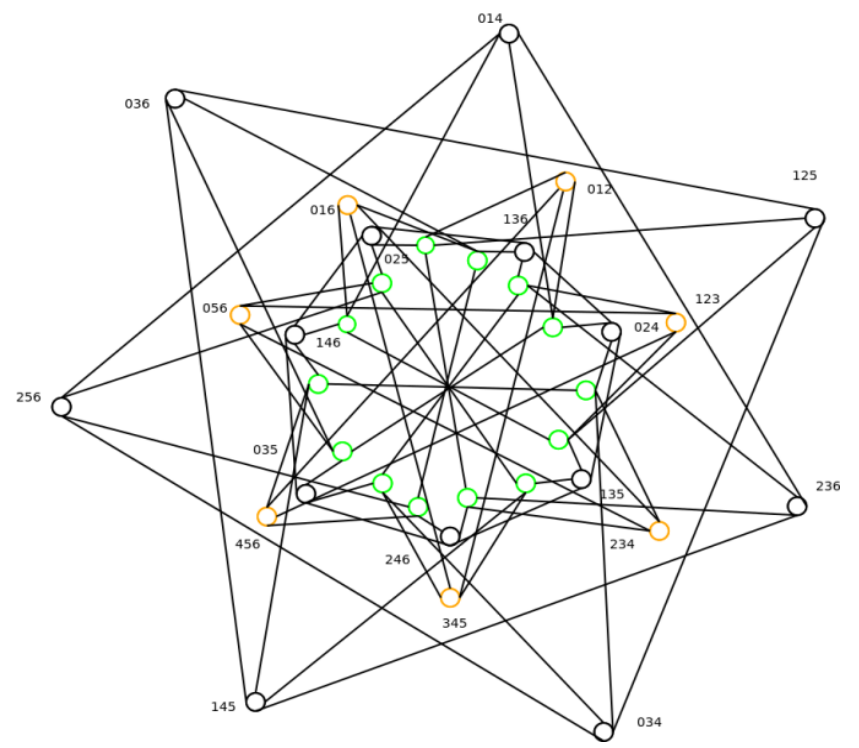

FIGURE III.

ODD GRAPH

Defeasible reasoning is suggested for supportingthe process of chosing appropriate help materials.

Tests results are often collected in different time periods. Only real experience indicates which questions and help materials are contributing for better learning. Usually there is a need for changing original preferences of recommendations which calls for involvement of techniques for ranking alternatives. In order to see the effect of all available data we suggest application of aggregating operators as described in [12]. Another approach is presented in [10].

If a student has a problem with answering a single question in test using the presented graphs is quite sufficient. If however a student provides wrong answers to two or more than two questions then the theory of weak orderings can be applied. One should keep in mind here that obtaining real ranking of preferences (in our case which test should be suggested first and the order in which the ones should follow) requires use of the tool for some time.

\section{CONCLUSION}

The main idea in this paper is to illustrate a model of a toll showing whether apparent lack of knowledge is related to intrinsic or explicit terms and concepts. The tool will support development of improved lectures by emphasizing where students experience problems in learning that subject. In addition it allows implementation on mobile devices. This will help lectures monitoring some learning activities during face to face teaching as well as analyzing results from automated tests taken by students in both controlled environment and on their spare time.

\section{REFERENCES}

[1] H. L. Andrade, and G. L. Cizek, eds., Handbook of Formative Assessment. New York, NY: Routledge, 2010.

[2] G. Antoniou, N. Dimaresis, and G. Governatori, "A modal and deontic defeasible reasoning system for modelling policies and multi-agent systems," Expert Systems with Applications, vol. 36, pp. 4125-4134, 2009 .

[3] N. Bassiliades, G. Antoniou, and I. Vlahavas, "A Defeasible Logic Reasoner for the Semantic Web," LNCS 3323, pp. 49-64, 2004.

[4] N. L. Biggs, "Algebraic Graph Theory," 2nd ed. Cambridge, England: Cambridge University Press, 1993.

[5] K. P. Bogart, "Some social sciences applications of ordered sets". In: I. Rival, Editor, Ordered Sets, Reidel, Dordrecht, pp. 759-787, 1982.

[6] B. Bollobas, "Extremal Graph Theory", Dover, 2004.

[7] D. C. Briggs, A. C. Alonzo, C., Schwab, and M. Wilson, "Diagnostic assessment with ordered multiple choice items", Educational Assessment, vol. 11 , no. $1,2006$.

[8] C. Chang, and K. Tseng, "Use and performances of Web-based portfolio assessment," British Journal of Educational Technology, vol. 40, No. 2, 2009.

[9] J. L. Gross and J. Yellen, Handbook of Graph Theory. CRC PressINC, 2004.

[10] D. Dubois and H. Prade, "A review of fuzzy sets aggregation connectives," Information Science, vol. 36, pp. 85-121, 1985.

[11] M. Kneser, "Aufgabe 360", Jahresbericht der Deutschen MathematikerVereinigung, 2. Abteilung 58: 27, 1955.

[12] J.-L. Marichal, "On Sugeno integral as an aggregation function," Fuzzy Sets Systems, vol. 114, pp. 347-366, 2000.

[13] J. H. McMillan, Classroom Assessment: Principles and Practices for Effective Standards-Based Instruction. New York, NY: Pearson, 2011.

[14] M. Rohaninezhad, S. Mo. Arif, and S. A. M. Noah, "A grounder for SPINdle defeasible logic reasoner," Expert Systems with Applications, vol. 42, pp. 7098-7109, 2015.

[15] A. Rupp, J. Templin, and R. Henson, Diagnostic Measurement: Theory, Methods, and Applications. New York, NY: Guilford Press, 2010.

[16] B. Zimmerman, and D. Schunk, eds., Handbook of Self-regulation of Learning and Performances. New York, NY: Routledge, 2011. 